\title{
HARDY TYPE INEQUALITIES WITH LOGARITHMIC AND POWER WEIGHTS FOR A SPECIAL FAMILY OF NON-CONVEX DOMAINS
}

\author{
R.G. NASIBULliN, A.M. TUKHVATULLINA
}

\begin{abstract}
In the present work we obtain variational Hardy type inequalities with power and logarithmic weights which are generalizations of the corresponding inequalities given earlier in the papers by M. Hoffmann-Ostenhof, T. Hoffmann-Ostenhof, A. Laptev, and J. Tidblom. We formulate and prove inequalities for arbitrary domains, and then we substantially simplify them for the class of convex domains and a special family of nonconvex domains.
\end{abstract}

Keywords: Hardy type inequalities, convex domains, regular domains, distance function, iteration of logarithms

Mathematics Subject Classification: 26D15.

1. Introduction. Variational inequalities is an important tool for solving problems of mathematical physics. In the present work we consider Hardy type variational inequalities. Hardy inequalities are used in the theory of degenerating elliptic partial differential equations, spectral theory, nonlinear analysis, and interpolation theory. For instance, in paper [1], Yu.A. Dubinski showed that a correct formulation of Poisson problem is equivalent to the validity of an appropriate Hardy inequality. Applications of Hardy type inequalities were also described in the works of A. Laptev, T. Weidl, A. Balinski, A. Sobolev, M. Solomyak, E. Davies [2]-[6].

A lot of works are devoted to studying and proving Hardy type inequalities, in particular, recent papers of F.G. Avkhadiev, K.-J. Wirths, E.B. Davies, M. Marcus, H. Brezis, M. HoffmannOstenhof, T. Hoffmann-Ostenhof, A. Laptev, J. Tidblom, G. Barbatis, S. Filippas, A. Tertikas [4]-[16].

The Hardy type inequality proven by J. Tidblom in paper [15] for an arbitrary domain $\Omega \subset \mathbb{R}^{n}(n \geq 2)$ and an arbitrary function $u \in W_{0}^{1, p}(\Omega)(p>1)$ reads as follows,

$$
\int_{\Omega}|\nabla u(x)|^{p} d x \geq \frac{c_{p} \sqrt{\pi} \Gamma\left(\frac{n+p}{2}\right)}{\Gamma\left(\frac{p+1}{2}\right) \Gamma\left(\frac{n}{2}\right)}\left(\int_{\Omega}|u(x)|^{p} \int_{\mathbb{S}^{n-1}} \frac{d \omega(\nu) d x}{\rho_{\nu}^{p}(x)}+(p-1)\left(\frac{\left|\mathbb{S}^{n-1}\right|}{n}\right)^{\frac{p}{n}} \int_{\Omega} \frac{|u(x)|^{p}}{\left|\Omega_{x}\right|^{\frac{p}{n}}} d x\right),
$$

where $\Omega_{x}:=\{y \in \Omega: x+t(y-x) \in \Omega, \forall t \in[0,1]\},\left|\Omega_{x}\right|$ is the measure of the domain $\Omega_{x}$, $\rho_{\nu}(x)$ is the distance from a point $x \in \Omega$ to the boundary of the domain $\Omega$ measured in the direction of a vector $\nu \in \mathbb{S}^{n-1},\left|\mathbb{S}^{n-1}\right|$ is the area of the unit sphere $\mathbb{S}^{n-1}$ in space $\mathbb{R}^{n}, d \mathbb{S}^{n-1}(\nu)$ is the element of surface measure on the unit sphere $\mathbb{S}^{n-1}, d \omega(\nu)=\frac{d \mathbb{S}^{n-1}(\nu)}{\left|\mathbb{S}^{n-1}\right|}, c_{p}=\left(\frac{p-1}{p}\right)^{p}$.

R.G. Nasibullin, A.M. Tukhvatullina, Hardy type inequalities with logarithmic And POWER WEIGHTS FOR A SPECIAL FAMILY OF NON-CONVEX DOMAIN.

(C) Nasibullin R.G., Tukhvatullina A.M. 2013.

The work is supported by Russian Foundation for Basic Researches (grant No. 11-01-00762).

Submitted March 30, 2012. 
We note that inequality (1) is a generalization for an arbitrary $p>1$ of an appropriate inequality proven by M. Hoffmann-Ostenhof, T. Hoffmann-Ostenhof, A. Laptev in paper [14] for $p=2$. In the present work we generalize inequality (1) for the functions in the space $C_{0}^{\infty}(\Omega)$ as follows,

$$
\begin{gathered}
\int_{\Omega}|\nabla u(x)|^{p} \int_{\mathbb{S}^{n-1}} \frac{|\cos (\nu, \nabla u)|^{p}}{\rho_{\nu}^{s-p}(x)} d \omega(\nu) d x \geq \\
\geq a(p, s) \int_{\Omega}|u(x)|^{p} \int_{\mathbb{S}^{n-1}} \frac{d \omega(\nu) d x}{\rho_{\nu}^{s}(x)}+a(p, s)(p-1)\left(\frac{\left|\mathbb{S}^{n-1}\right|}{n}\right)^{\frac{s}{n}} \int_{\Omega} \frac{|u(x)|^{p}}{\left|\Omega_{x}\right|^{\frac{s}{n}}} d x,
\end{gathered}
$$

where $p \geq s \geq 1$ and $a(p, s)=\left(\frac{s-1}{p}\right)^{p}$.

It is obvious that for $s=p$ the latter inequality becomes inequality (1), while as $s=p=2$ it casts into the inequality proven by M. Hoffmann-Ostenhof, T. Hoffmann-Ostenhof, A. Laptev in [14].

In paper [15] it was also shown that in the case of a convex domain $\Omega \subset \mathbb{R}^{n}$ inequality (1) can be substantially simplified. Namely, (1) can rewritten as

$$
\int_{\Omega}|\nabla u(x)|^{p} d x \geq c_{p} \int_{\Omega} \frac{|u(x)|^{p}}{\delta^{p}(x)} d x+\frac{c_{p}(p-1) \sqrt{\pi} \Gamma\left(\frac{n+p}{2}\right)}{\Gamma\left(\frac{p+1}{2}\right) \Gamma\left(\frac{n}{2}\right)}\left(\frac{\left|\mathbb{S}^{n-1}\right|}{n|\Omega|}\right)^{\frac{p}{n}} \int_{\Omega}|u(x)|^{p} d x .
$$

Our inequality (2) can be also substantially simplified for convex domains,

$$
\int_{\Omega} \frac{|\nabla u(x)|^{p}}{\delta^{s-p}(x)} \geq a(p, s) \frac{\Gamma\left(\frac{s+1}{2}\right) \Gamma\left(\frac{n}{2}\right)}{\sqrt{\pi} \Gamma\left(\frac{n+s}{2}\right)} \int_{\Omega} \frac{|u(x)|^{p}}{\delta^{s}(x)}+a(p, s)(p-1)\left(\frac{\left|\mathbb{S}^{n-1}\right|}{n|\Omega|}\right)^{\frac{s}{n}} \int_{\Omega}|u(x)|^{p} d x .
$$

In the present work we also provide a special class of non-convex domains (see [5], [21], [22], and [23]) for that analogues of inequality (4) hold true.

In the concluding part of the paper we establish logarithmic inequalities being analogues of those in [14] and [16]. The inequality proven in [14] for a convex domain $\Omega \subset \mathbb{R}^{n}$ reads as

$$
\begin{gathered}
\int_{\Omega}|\nabla u(x)|^{2} d x \geq \frac{1}{4} \int_{\Omega} \frac{|u(x)|^{2}}{\delta(x)^{2}}\left(1+\frac{1}{(1-\ln (\alpha \delta(x) / D))^{2}}\right) d x+ \\
+\frac{n^{(n-2) / n} s_{n-1}^{2 / n} \ln ^{2}(\alpha / 2)}{4(1-\ln (\alpha / 2))^{2}} \frac{1}{|\Omega|^{2 / n}} \int_{\Omega}|u(x)|^{2} d x .
\end{gathered}
$$

A feature of our logarithmic inequalities is the presence of iteration of logarithms and exponents. The examples of employing logarithms can be found in works [11, [12, [14], [17]-[19], and [20]. We observe that a generalization of inequality (5) by help of embedded logarithms was obtained earlier in [16]. But we obtain the inequality with another logarithmic weight for a class of regular domains. Namely, we show that for an arbitrary domain $\Omega \subset \mathbb{R}^{n}$ with constant of regularity $c$ and and an arbitrary function $u \in C_{0}^{\infty}(\Omega)$ inequality

$$
\begin{gathered}
\int_{\Omega}|\nabla u(x)|^{2} d x \geq \frac{n}{2 c^{2}} \int_{\Omega} \frac{|u(x)|^{2}}{\delta^{2}(x)}\left(1+\sum_{i=0}^{k} \varphi_{0}^{2}\left(\frac{\alpha \delta}{D}, e_{k}\right) \cdot \ldots \cdot \varphi_{i}^{2}\left(\frac{\alpha \delta}{D}, e_{k}\right)\right) d x+ \\
+\left[1-\sum_{i=0}^{k} \varphi_{0}\left(\frac{\alpha}{2}, e_{k}\right) \cdot \ldots \cdot \varphi_{i}\left(\frac{\alpha}{2}, e_{k}\right)\right]^{2} \frac{n^{(n-2) / 2}}{4} s_{n-1}^{2 / n} \frac{1}{|\Omega|^{2 / n}} \int_{\Omega}|u(x)|^{2} d x
\end{gathered}
$$


holds true, where

$$
\begin{gathered}
e_{0}=1, \quad e_{k+1}=\exp e_{k} ; \quad \ln _{0} x=x, \quad \ln _{k+1}(x)=\ln \ln _{k}(x), \\
\varphi_{i}\left(x, e_{k}\right)=\frac{1}{\left(e_{k}-\ln x\right) \ln \left(e_{k}-\ln x\right) \cdot \ldots \cdot \ln _{i}\left(e_{k}-\ln x\right)}, i \leqslant k .
\end{gathered}
$$

The latter statement for $k=0$ yields (5).

2. One-dimensional inequalities. Let us prove one-dimensional inequalities which will be employed later in the proof of inequalities in the multi-dimensional case. We introduce required definitions and notations.

Let $f$ be defined and differentiable on $(0, b]$ for $b>0$. Following J. Tidblom (see [15]), we shall say that $f \in \Phi_{s}(0, b)$ if $f$ is a real function and there exists a constant $C=C(f)$ such that

$$
\sup _{0<t \leqslant b}\left(t^{s-1}|f(t)|+t^{s}\left|f^{\prime}(t)\right|\right) \leqslant C, \quad s>1 .
$$

It is easy to see that condition (3) is equivalent to two conditions,

$$
\exists C_{1}=C_{1}(f): t^{s-1}|f(t)| \leqslant C_{1}, 0<t \leqslant b,
$$

and

$$
\exists C_{2}=C_{2}(f): t^{s}\left|f^{\prime}(t)\right| \leqslant C_{2}, 0<t \leqslant b .
$$

Lemma 1. Suppose $u \in C^{1}[0, b], b>0, u(0)=0$ and $f \in \Phi_{s}(0, b)$. Then as $p \geq s>1$, the inequality

holds true.

$$
\int_{0}^{b} \frac{\left|u^{\prime}(t)\right|^{p}}{t^{s-p}} d t \geq \frac{1}{p^{p}} \frac{\left.\left.\left|\int_{0}^{b} f^{\prime}(t)\right| u(t)\right|^{p} d t\right|^{p}}{\left(\int_{0}^{b}|f(t)-f(b)|^{\frac{p}{p-1}} t^{\frac{s-p}{p-1}}|u(t)|^{p}\right)^{p-1}}
$$

Proof. By the hypothesis of the lemma, for the function $u(t)$ we have

$$
\exists M>0:|u(t)| \leqslant \int_{0}^{t}\left|u^{\prime}(x)\right| d x \leqslant M t, \forall t \in(0, b] .
$$

Taking into consideration condition (8) and inequality $p \geq s$, we obtain

$$
\left|f^{\prime}(t)\right||u(t)|^{p} \leqslant\left|f^{\prime}(t)\right| M^{p} t^{p} \leqslant\left|f^{\prime}(t)\right| t^{s} M^{p} t^{p-s} \leqslant C_{2} M^{p} b^{p-s} .
$$

Thus,

$$
\int_{0}^{b} f^{\prime}(t)|u(t)|^{p} d t \leqslant+\infty
$$

We also observe that

$$
\begin{gathered}
f(0)|u(0)|^{p}=\lim _{t \rightarrow 0} f(t)|u(t)|^{p}=\lim _{t \rightarrow 0} f(t) t^{s-1} \frac{|u(t)|^{p}}{t^{s-1}} \leqslant \\
\leqslant \lim _{t \rightarrow 0} C_{1} \frac{|u(t)|^{p}}{t^{s-1}} \leqslant C_{1} \lim _{t \rightarrow 0} \frac{M^{p-1} t^{p-1}|u(t)|}{t^{s-1}} \leqslant \\
\leqslant C_{1} \lim _{t \rightarrow 0} M^{p-1} t^{p-s}|u(t)| \leqslant C_{1} M^{p-1} b^{p-s}|u(0)|=0 .
\end{gathered}
$$

For an arbitrary constant $c$ we get

$$
\left.|(f(b)-c)| u(b)\right|^{p}-\int_{0}^{b} f^{\prime}(t)|u(t)|^{p} d t|=| \int_{0}^{b}(f(t)-c)\left(|u(t)|^{p}\right) d t \mid=
$$




$$
\begin{gathered}
=\frac{p}{2}\left|\int_{0}^{b}(f(t)-c)\left(u^{\frac{p}{2}-1} \bar{u}^{\frac{p}{2}} u^{\prime}+\bar{u}^{\frac{p}{2}-1} u^{\frac{p}{2}} \bar{u}^{\prime}\right) d t\right| \leqslant \\
\leqslant p \int_{0}^{b}|f(t)-c||u|^{p-1}\left|u^{\prime}(t)\right| d t=p \int_{0}^{b}\left[|f(t)-c|^{\frac{p}{p-1}} t^{\frac{s-p}{p-1}} u^{p}\right]^{\frac{p-1}{p}}\left[\frac{\left|u^{\prime}(t)\right|^{p}}{t^{s-p}}\right]^{\frac{1}{p}} d t \leqslant \\
\leqslant p\left(\int_{0}^{b}|f(t)-c|^{\frac{p}{p-1}} t^{\frac{s-p}{p-1}}|u|^{p} d t\right)^{\frac{p-1}{p}}\left(\int_{0}^{b} \frac{\left|u^{\prime}(t)\right|^{p}}{t^{s-p}} d t\right)^{\frac{1}{p}} .
\end{gathered}
$$

Substituting $c=f(b)$ and taking $p$-th power of both sides of the inequality, we obtain the desired estimate.

Remark. In order the integral in the first factor of the latter inequality to have no singularities, one needs restrictions for $s$ and $p$. As $p \geq s \geq 1$, this integral has no singularities due to the inequality

$$
\frac{s-p}{p-1}+p \geq 0
$$

since $|u(t)| \leqslant M t$.

Let us give some corollaries of Lemma 1.

Corollary 1. Suppose $u(t)$ satisfies the hypothesis of the previous lemma and $f(t)=\frac{t^{1-s}}{1-s}$. Then the inequality

$$
\int_{0}^{b} \frac{\left|u^{\prime}(t)\right|^{p}}{t^{s-p}} d t \geq a(p, s) \frac{\left|\int_{0}^{b} \frac{|u(t)|^{p}}{t^{s}} d t\right|^{p}}{\left(\int_{0}^{b}\left|t^{1-s}-b^{1-s}\right|^{\frac{p}{p-1}} t^{\frac{s-p}{p-1}}|u(t)|^{p}\right)^{p-1}}
$$

holds true, where $a(p, s)=\left(\frac{s-1}{p}\right)^{p}$.

Corollary 2. Let $u \in C^{1}[0, b], b>0, u(0)=0$. Then

$$
\int_{0}^{b} \frac{\left|u^{\prime}(t)\right|^{p}}{t^{s-p}} d t \geq a(p, s)\left(\int_{0}^{b}\left(\frac{p}{t^{s}}-(p-1)\left(t^{1-s}-b^{1-s}\right)^{\frac{p}{p-1}} t^{\frac{s-p}{p-1}}\right)|u(t)|^{p} d t\right) .
$$

Proof. The equation follows from Corollary 1 and a simple inequality

$$
\frac{A^{p}}{B^{p-1}} \geq p A-(p-1) B
$$

if we let

$$
A=\int_{0}^{b} \frac{|u(t)|^{p}}{t^{s}} d t
$$

and

$$
B=\int_{0}^{b}\left|t^{1-s}-b^{1-s}\right|^{\frac{p}{p-1}}|u(t)|^{p} d t
$$


Lemma 2. Suppose $u \in C_{0}^{\infty}(0,2 b), b>0$. Then we have

$$
\int_{0}^{2 b} \frac{\left|u^{\prime}(t)\right|^{p}}{\rho(t)^{s-p}} d t \geq a(p, s)\left(\int_{0}^{2 b}\left(\frac{p}{\rho^{s}(t)}-(p-1)\left(\rho^{1-s}-b^{1-s}\right)^{\frac{p}{p-1}} \rho^{\frac{s-p}{p-1}}(t)\right)|u(t)|^{p} d t\right),
$$

where

$$
\rho(t)=\operatorname{dist}(t, \mathbb{R} \backslash[0,2 b])=\min (t, 2 b-t) .
$$

Proof. We apply inequality (9) to a function $u \in C^{1}[b, 2 b]$ obeying $u(2 b)=0$. We have

$$
\int_{b}^{2 b} \frac{\left|u^{\prime}(t)\right|^{p}}{(2 b-t)^{s-p}} d t \geq a(p, s)\left(\int_{b}^{2 b}\left(\frac{p}{(2 b-t)^{s}}-(p-1)\left((2 b-t)^{1-s}-b^{1-s}\right)^{\frac{p}{p-1}}(2 b-t)^{\frac{s-p}{p-1}}\right)|u|^{p} d t\right) .
$$

Summing the obtained inequality with (9), we arrive at the statement of the lemma.

Theorem 1. Let $u \in C_{0}^{\infty}(a, b)$. Then we have

$$
\int_{a}^{b} \frac{\left|u^{\prime}(t)\right|^{p}}{\rho(t)^{s-p}} d t \geq a(p, s)\left(\int_{a}^{b} \frac{|u(t)|^{p}}{\rho^{s}(t)} d t-\frac{p-1}{\left(\frac{b-a}{2}\right)^{s}} \int_{a}^{b}|u(t)|^{p} d t\right)
$$

Proof. Without loss of generality, we take the segment $[0,2 b]$ as the interval of integration. The right hand side of the inequality in Lemma 2 can be rewritten as

$$
a(p, s)\left(\int_{0}^{2 b} \frac{|u(t)|^{p}}{\rho(t)^{s}} d t+\int_{0}^{2 b} \frac{p-1}{\rho(t)^{s}}\left(1-\left(1-\left(\frac{\rho(t)}{b}\right)^{s-1}\right)^{\frac{p}{p-1}}\right)|u(t)|^{p} d t\right) .
$$

Here we have employed the identity $s-\frac{p(s-1)}{p-1}+\frac{s-p}{p-1}=0$.

We note that $\rho(t) \leqslant b$. Hence,

$$
\begin{gathered}
\frac{1}{\rho(t)^{s}}\left(1-\left(1-\left(\frac{\rho(t)}{b}\right)^{s-1}\right)^{\frac{p}{p-1}}\right) \geq \frac{1}{\rho(t)^{s}}\left(1-\left(1-\left(\frac{\rho(t)}{b}\right)^{s-1}\right)\right)= \\
=\frac{1}{\rho(t) b^{s}} \geq \frac{1}{b^{s}} .
\end{gathered}
$$

It implies the statement of the theorem.

3. Multi-dimensional Hardy type inequalities for arbitrary open domains. In this section we provide a multi-dimensional analogue of the inequality in Theorem 1.

Let $\Omega$ be an open domain in $\mathbb{R}^{n}$. Following M. Hoffmann-Ostenhof, T. Hoffmann-Ostenhof, A. Laptev [14, we denote by $\tau_{\nu}(x)$ the distance between a point $x \in \Omega$ and the nearest point on the boundary $\partial \Omega$ in the direction of the vector $\nu \in \mathbb{S}^{n-1}$,

$$
\tau_{\nu}(x)=\min \{s>0: x+s \nu \in \Omega\} .
$$

We define $\rho_{\nu}(x)$ being the distance from the boundary of the domain measured in the direction of $\nu$ and $D_{\nu}(x)$ being the diameter of the domain in the direction of $\nu$ as follows,

$$
\rho_{\nu}(x)=\min \left\{\tau_{-\nu}(x), \tau_{\nu}(x)\right\}, D_{\nu}(x)=\tau_{\nu}(x)+\tau_{-\nu}(x) .
$$

Let

$$
\delta(x)=\inf _{\nu \in \mathbb{S}^{n-1}} \tau_{\nu}(x)=\operatorname{dist}(x, \partial \Omega), \Omega_{x}=\{y \in \Omega: x+t(y-x) \in \Omega, \forall t \in[0,1]\}
$$


Theorem 2. For an arbitrary open domain $\Omega \subset \mathbb{R}^{n}$ and an arbitrary function $u \in C_{0}^{\infty}(\Omega)$, the following Hardy type inequality

$$
\begin{gathered}
\int_{\Omega}|\nabla u(x)|^{p} \int_{\mathbb{S}^{n-1}} \frac{|\cos (\nu, \nabla u(x))|^{p}}{\rho_{\nu}^{s-p}(x)} d \omega(\nu) d x \geq \\
\geq a(p, s)\left[\int_{\Omega}|u(x)|^{p} \int_{\mathbb{S}^{n-1}} \frac{d \omega(\nu)}{\rho_{\nu}^{s}(x)} d x+(p-1)\left(\frac{\left|\mathbb{S}^{n-1}\right|}{n}\right)^{\frac{s}{n}} \int_{\Omega} \frac{|u(x)|^{p}}{\left|\Omega_{x}\right|^{s / n}} d x\right]
\end{gathered}
$$

holds true.

Proof. Using E.B. Davies' arguments (see [5]) and one-dimensional inequality (10), it is easy to get the following estimate,

$$
\int_{\Omega} \frac{\left|\partial_{\nu} u(x)\right|^{p}}{\rho_{\nu}(x)^{s-p}} d x \geq a(p, s) \int_{\Omega} \frac{|u(x)|^{p}}{\rho_{\nu}^{s}(x)} d t-a(p, s)(p-1) \int_{\Omega}\left(\frac{2}{D_{\nu}(x)}\right)^{s}|u(x)|^{p} d x .
$$

Employing the definition of the gradient, we have

$$
\left|\partial_{\nu} u(x)\right|=|\nu \cdot \nabla u(x)|=|\nabla u(x)||\cos (\nu, \nabla u(x))| .
$$

We integrate both sides of the inequality w.r.t. normal surface measure $\mathbb{S}^{n-1}$. We get

$$
\begin{gathered}
\int_{\Omega} \int_{\mathbb{S}^{n-1}} \frac{|\cos (\nu, \nabla u(x))|^{p}}{\rho_{\nu}(x)^{s-p}} d \omega(\nu)|\nabla u(x)|^{p} d x \geq \\
\geq a(p, s)\left(\int_{\Omega} \int_{\mathbb{S}^{n-1}}\left(\frac{1}{\rho_{\nu}^{s}(x)}-(p-1) \int_{\mathbb{S}^{n-1}}\left(\frac{2}{D_{\nu}(x)}\right)^{s} d \omega(\nu)\right)|u(x)|^{p} d x\right) .
\end{gathered}
$$

Due to [15], it is known that

$$
\int_{\mathbb{S}^{n-1}}\left(\frac{2}{D_{\nu}(x)}\right)^{s} d \omega(\nu) \geq\left(\frac{n\left|\Omega_{x}\right|}{\left|\mathbb{S}^{n-1}\right|}\right)^{-\frac{s}{n}}
$$

Hence, the inequality

$$
\begin{gathered}
\int_{\Omega}|\nabla u(x)|^{p} \int_{\mathbb{S}^{n-1}} \frac{|\cos (\nu, \nabla u(x))|^{p}}{\rho_{\nu}^{s-p}(x)} d \omega(\nu) d x \geq \\
\geq a(p, s)\left[\int_{\Omega}|u(x)|^{p} \int_{\mathbb{S}^{n-1}} \frac{d \omega(\nu)}{\rho_{\nu}^{s}(x)} d x+(p-1)\left(\frac{\left|\mathbb{S}^{n-1}\right|}{n}\right)^{\frac{s}{n}} \int_{\Omega} \frac{|u(x)|^{p}}{\left|\Omega_{x}\right|^{s / n}} d x\right]
\end{gathered}
$$

holds true. The proof is complete.

4. Hardy type inequalities for convex domains. In Section 2 we have proven that for an arbitrary domain $\Omega \subset \mathbb{R}^{n}$ and a function $u \in C_{0}^{\infty}(\Omega)$ the following Hardy type inequality

$$
\begin{gathered}
\int_{\Omega}|\nabla u(x)|^{p} \int_{\mathbb{S}^{n-1}} \frac{|\cos (\nu, \nabla u(x))|^{p}}{\rho_{\nu}^{s-p}(x)} d \omega(\nu) d x \geq \\
\geq a(p, s) \int_{\Omega}|u(x)|^{p} \int_{\mathbb{S}^{n-1}} \frac{d \omega(\nu) d x}{\rho_{\nu}^{s}(x)}+a(p, s)(p-1)\left(\frac{\left|\mathbb{S}^{n-1}\right|}{n}\right)^{\frac{s}{n}} \int_{\Omega} \frac{|u(x)|^{p}}{\left|\Omega_{x}\right|^{\frac{s}{n}}} d x
\end{gathered}
$$

holds true. As it will be shown in the next theorem, in the case of a convex domain $\Omega \subset \mathbb{R}^{n}$ inequality (11) can be substantially simplified. 
Theorem 3. Let $\Omega \subset \mathbb{R}^{n}$ be an arbitrary convex domain, $u \in C_{0}^{\infty}(\Omega)$ is an arbitrary function. Then the inequality

$$
\int_{\Omega} \frac{|\nabla u(x)|^{p}}{\delta^{s-p}(x)} \geq a(p, s) \frac{\Gamma\left(\frac{s+1}{2}\right) \Gamma\left(\frac{n}{2}\right)}{\sqrt{\pi} \Gamma\left(\frac{n+s}{2}\right)} \int_{\Omega} \frac{|u(x)|^{p}}{\delta^{s}(x)}+a(p, s)(p-1)\left(\frac{\left|\mathbb{S}^{n-1}\right|}{n|\Omega|}\right)^{\frac{s}{n}} \int_{\Omega}|u(x)|^{p} d x
$$

holds true.

Proof. For an arbitrary convex domain $\Omega \subset \mathbb{R}^{n}$ we estimate from above the internal integral in the left hand side of (11),

$$
\begin{gathered}
\int_{\mathbb{S}^{n-1}} \frac{|\cos (\nu, \nabla u(x))|^{p}}{\rho_{\nu}^{s-p}(x)} d \omega(\nu)=\int_{\mathbb{S}^{n-1}} \frac{|\cos (\nu, \nabla u(x))|^{p} \rho_{\nu}^{p}(x)}{\rho_{\nu}^{s}(x)} d \omega(\nu) \leqslant \\
\leqslant \int_{\mathbb{S}^{n-1}} \frac{|\cos (\nu, \nabla u(x))|^{p} \rho_{\nu}^{p}(x)}{\delta^{s}(x)} d \omega(\nu)=\int_{\mathbb{S}^{n-1}} \frac{|\cos (\nu, e)|^{p} \rho_{\nu}^{p}(x)}{\delta^{s}(x)} d \omega(\nu) \leqslant \\
\leqslant \int_{\mathbb{S}^{n-1}} \frac{\delta^{p}(x)}{\delta^{s}(x)} d \omega(\nu)=\int_{\mathbb{S}^{n-1}} \frac{d \omega(\nu)}{\delta^{s-p}(x)}=\frac{1}{\delta^{s-p}(x)},
\end{gathered}
$$

where $e=\nu_{0} \in \mathbb{S}^{n-1}: \tau_{\nu_{0}}(x)=\delta(x)$.

In the latter chain of relations we have employed the inequality $|\cos (\nu, e)| \rho_{\nu}(x) \leqslant \delta(x)$ that is valid for all points $x \in \Omega$ due to obvious geometric arguments.

Thus, for the left hand side of inequality (11) we obtain the estimate

$$
\int_{\Omega}|\nabla u(x)|^{p} \int_{\mathbb{S}^{n-1}} \frac{|\cos (\nu, \nabla u(x))|^{p}}{\rho_{\nu}^{s-p}(x)} d \omega(\nu) d x \leqslant \int_{\Omega} \frac{|\nabla u(x)|^{p}}{\delta^{s-p}(x)} .
$$

Applying inequality $|\cos (\nu, e)| \rho_{\nu}(x) \leqslant \delta(x)$ to the internal integral of the first term in the right hand side of inequality (11), we obviously obtain

$$
\int_{\mathbb{S}^{n-1}} \frac{d \omega(\nu)}{\rho_{\nu}^{s}(x)} \geq \int_{\mathbb{S}^{n-1}} \frac{|\cos (\nu, e)|^{s} d \omega(\nu)}{\delta^{s}(x)}=\frac{1}{\delta^{s}(x)} \int_{\mathbb{S}^{n-1}}|\cos (\nu, e)|^{s} d \omega(\nu) .
$$

The latter integral can be easily calculated by the change of variables,

$$
\int_{\mathbb{S}^{n-1}}|\cos (\nu, e)|^{s} d \omega(\nu)=\frac{\Gamma\left(\frac{s+1}{2}\right) \Gamma\left(\frac{n}{2}\right)}{\sqrt{\pi} \Gamma\left(\frac{n+s}{2}\right)} .
$$

Hence, we have the lower estimate for the first term in the right hand side of inequality (11),

$$
a(p, s) \int_{\Omega}|u(x)|^{p} \int_{\mathbb{S}^{n-1}} \frac{d \omega(\nu) d x}{\rho_{\nu}^{s}(x)} \geq a(p, s) \frac{\Gamma\left(\frac{s+1}{2}\right) \Gamma\left(\frac{n}{2}\right)}{\sqrt{\pi} \Gamma\left(\frac{n+s}{2}\right)} \int_{\Omega} \frac{|u(x)|^{p}}{\delta^{s}(x)} .
$$

Employing the identity $\Omega_{x}=\Omega$, which is obvious for convex domains, we obtain easily the identity for the second term in the right hand side of inequality (11),

$$
a(p, s)(p-1)\left(\frac{\left|\mathbb{S}^{n-1}\right|}{n}\right)^{\frac{s}{n}} \int_{\Omega} \frac{|u(x)|^{p}}{\left|\Omega_{x}\right|^{\frac{s}{n}}} d x=a(p, s)(p-1)\left(\frac{\left|\mathbb{S}^{n-1}\right|}{n|\Omega|}\right)^{\frac{s}{n}} \int_{\Omega}|u(x)|^{p} d x .
$$

The proof is complete. 
Comparing inequalities (11) and (12), we pose a question whether there exist non-convex domains satisfying analogues of inequality (12) proven in Theorem 3 for convex domains? We give the positive answer for this question and provide a special class of non-convex domains for which analogues of inequality 12 exist.

5. Hardy type inequalities for non-convex regular domains. Following E.B. Davies [5], we define a pseudo-distance $m(x)$ from a point $x$ to the boundary of the domain $\Omega$,

$$
\frac{1}{m^{2}(x)}:=\frac{1}{\left|\mathbb{S}^{n-1}\right|} \int_{\mathbb{S}^{n-1}} \frac{d \mathbb{S}^{n-1}(\nu)}{\tau_{\nu}^{2}(x)}
$$

We introduce the notion of a regular domain in space $\mathbb{R}^{n}$. We shall say that the domain $\Omega \subset \mathbb{R}^{n}$ is regular if there exists a finite constant $c>0$ such that

$$
\delta(x) \leqslant m(x) \leqslant c \delta(x) \quad \forall x \in \Omega .
$$

We shall call $c$ a regularity constant for the domain $\Omega$.

As it will be shown in the next theorem, for regular domains it is possible to obtain an inequality similar to inequality (12) proven in Theorem 3 for convex domains.

Theorem 4. Suppose $\Omega \subset \mathbb{R}^{n}$ is an arbitrary regular domains with the constant of regularity $c, u \in C_{0}^{\infty}(\Omega)$ is an arbitrary function. Then the inequality

$$
\begin{gathered}
\frac{D_{p}(\Omega) \Gamma\left(\frac{p+1}{2}\right) \Gamma\left(\frac{n}{2}\right)}{\sqrt{\pi} \Gamma\left(\frac{n+p}{2}\right)} \int_{\Omega} \frac{|\nabla u(x)|^{p}}{\delta^{s}(x)} d x \geq \\
\geq \frac{a(p, s) 2^{s / 2}}{c^{s}} \int_{\Omega} \frac{|u(x)|^{p}}{\delta^{s}(x)} d x+a(p, s)(p-1)\left(\frac{\left|\mathbb{S}^{n-1}\right|}{n|\Omega|}\right)^{\frac{s}{n}} \int_{\Omega}|u(x)|^{p} d x
\end{gathered}
$$

holds true, where $D_{p}(\Omega):=\sup _{\nu \in \mathbb{S}^{n-1}} \rho_{\nu}^{p}(x), x \in \Omega$.

Proof. For an arbitrary regular domain $\Omega \subset \mathbb{R}^{n}$ with the constant of regularity $c$ we estimate from above the internal integral in the left hand side of inequality (11),

$$
\begin{gathered}
\int_{\mathbb{S}^{n-1}} \frac{|\cos (\nu, \nabla u(x))|^{p}}{\rho_{\nu}^{s-p}(x)} d \omega(\nu)=\int_{\mathbb{S}^{n-1}} \frac{|\cos (\nu, \nabla u(x))|^{p} \rho_{\nu}^{p}(x)}{\rho_{\nu}^{s}(x)} d \omega(\nu) \leqslant \\
\leqslant \int_{\mathbb{S}^{n-1}} \frac{|\cos (\nu, \nabla u(x))|^{p} \rho_{\nu}^{p}(x)}{\delta^{s}(x)} d \omega(\nu)=\int_{\mathbb{S}^{n-1}} \frac{|\cos (\nu, e)|^{p} \rho_{\nu}^{p}(x)}{\delta^{s}(x)} d \omega(\nu) \leqslant \\
\leqslant \frac{D_{p}(\Omega)}{\delta^{s}(x)} \int_{\mathbb{S}^{n-1}}|\cos (\nu, e)|^{p} d \omega(\nu)=\frac{D_{p}(\Omega) \Gamma\left(\frac{p+1}{2}\right) \Gamma\left(\frac{n}{2}\right)}{\delta^{s}(x) \sqrt{\pi} \Gamma\left(\frac{n+p}{2}\right)},
\end{gathered}
$$

where $e=\nu_{0} \in \mathbb{S}^{n-1}: \tau_{\nu_{0}}(x)=\delta(x), D_{p}(\Omega):=\sup _{\nu \in \mathbb{S}^{n-1}} \rho_{\nu}^{p}(x), x \in \Omega$.

Thus, for the left hand side of inequality (11) we obtain the estimate

$$
\int_{\Omega}|\nabla u(x)|^{p} \int_{\mathbb{S}^{n-1}} \frac{|\cos (\nu, \nabla u(x))|^{p}}{\rho_{\nu}^{s-p}(x)} d \omega(\nu) d x \leqslant \frac{D_{p}(\Omega) \Gamma\left(\frac{p+1}{2}\right) \Gamma\left(\frac{n}{2}\right)}{\sqrt{\pi} \Gamma\left(\frac{n+p}{2}\right)} \int_{\Omega} \frac{|\nabla u(x)|^{p}}{\delta^{s}(x)} .
$$


To estimate the integral integral of the first term in the right hand side of inequality (11), we employ a result proven in [21] for regular domains,

$$
\int_{\mathbb{S}^{n-1}} \frac{d \omega(\nu)}{\rho_{\nu}^{s}(x)} \geq \frac{2^{s / 2}}{m^{s}(x)}
$$

Taking into consideration the inequality

$$
m(x) \leqslant c \delta(x)
$$

being valid for all point $x$ in the domain $\Omega$ regular with constant $c$, we arrive easily to the inequality

$$
\int_{\mathbb{S}^{n-1}} \frac{d \omega(\nu)}{\rho_{\nu}^{s}(x)} \geq \frac{2^{s / 2}}{c^{s} \delta^{s}(x)}
$$

Thus,

$$
a(p, s) \int_{\Omega}|u(x)|^{p} \int_{\mathbb{S}^{n-1}} \frac{d \omega(\nu) d x}{\rho_{\nu}^{s}(x)} \geq \frac{a(p, s) 2^{s / 2}}{c^{s}} \int_{\Omega} \frac{|u(x)|^{p}}{\delta^{s}(x)} d x .
$$

Employing the obvious inequality $\left|\Omega_{x}\right| \leqslant|\Omega|$ being valid for any domain $\Omega \subset \mathbb{R}^{n}$, we obtain easily the estimate for the second term in the right hand side of inequality (11),

$$
a(p, s)(p-1)\left(\frac{\left|\mathbb{S}^{n-1}\right|}{n}\right)^{\frac{s}{n}} \int_{\Omega} \frac{|u(x)|^{p}}{\left|\Omega_{x}\right|^{\frac{s}{n}}} d x \geq a(p, s)(p-1)\left(\frac{\left|\mathbb{S}^{n-1}\right|}{n|\Omega|}\right)^{\frac{s}{n}} \int_{\Omega}|u(x)|^{p} d x .
$$

The proof is complete.

6. Hardy type inequalities with logarithmic weights for regular domains and functions in space $H_{0}^{1}$. Let

$$
f(t)=-\frac{1}{t}+\frac{1}{t\left(e-\ln \frac{\alpha t}{D}\right)}+\frac{1}{t\left(e-\ln \frac{\alpha t}{D}\right) \ln \left(e-\ln \frac{\alpha t}{D}\right)}, \quad 0<t<D / 2,
$$

where $D=\operatorname{diam} \Omega$ and $0<\alpha \leqslant 2$.

Then the following identity

$$
\begin{gathered}
2 f^{\prime}\left(\rho_{\nu}\right)-f^{2}\left(\rho_{\nu}\right)=\frac{2}{\rho_{\nu}^{2}}-\frac{2}{\rho_{\nu}^{2}\left(e-\ln \frac{\alpha \rho_{\nu}}{D}\right)}+\frac{2}{\rho_{\nu}^{2}\left(e-\ln \frac{\alpha \rho_{\nu}}{D}\right)^{2}}-\frac{2}{\rho_{\nu}^{2}\left(e-\ln \frac{\alpha \rho_{\nu}}{D}\right) \ln \left(e-\ln \frac{\alpha \rho_{\nu}}{D}\right)}+ \\
+\frac{2}{\rho_{\nu}^{2}\left(e-\ln \frac{\alpha \rho_{\nu}}{D}\right)^{2} \ln \left(e-\ln \frac{\alpha \rho_{\nu}}{D}\right)}+\frac{1}{\rho_{\nu}^{2}\left(e-\ln \frac{\alpha \rho_{\nu}}{D}\right)^{2} \ln ^{2}\left(e-\ln \frac{\alpha \rho_{\nu}}{D}\right)}- \\
\quad-\frac{1}{\rho_{\nu}^{2}}-\frac{1}{\rho_{\nu}^{2}\left(e-\ln \frac{\alpha \rho_{\nu}}{D}\right)^{2}}-\frac{1}{\rho_{\nu}^{2}\left(e-\ln \frac{\alpha \rho_{\nu}}{D}\right)^{2} \ln ^{2}\left(e-\ln \frac{\alpha \rho_{\nu}}{D}\right)}+ \\
+\frac{2}{\rho_{\nu}^{2}\left(e-\ln \frac{\alpha \rho_{\nu}}{D}\right)}+\frac{2}{\rho_{\nu}^{2}\left(e-\ln \frac{\alpha \rho_{\nu}}{D}\right) \ln \left(e-\ln \frac{\alpha \rho_{\nu}}{D}\right)}-\frac{1}{\rho_{\nu}^{2}\left(e-\ln \frac{\alpha \rho_{\nu}}{D}\right)^{2} \ln \left(e-\ln \frac{\alpha \rho_{\nu}}{D}\right)}= \\
=\frac{1}{\rho_{\nu}^{2}}+\frac{1}{\rho_{\nu}^{2}\left(e-\ln \frac{\alpha \rho_{\nu}}{D}\right)^{2}}+\frac{1}{\rho_{\nu}^{2}\left(e-\ln \frac{\alpha \rho_{\nu}}{D}\right)^{2} \ln ^{2}\left(e-\ln \frac{\alpha \rho_{\nu}}{D}\right)}
\end{gathered}
$$

holds true. The estimate

$$
\begin{aligned}
2 f\left(\rho_{\nu}\right) f\left(D_{\nu} / 2\right) & -f^{2}\left(D_{\nu} / 2\right)=\frac{4}{\rho_{\nu} D_{\nu}}\left[1-\frac{1}{e-\ln \frac{\alpha \rho_{\nu}}{D}}-\frac{1}{\left(e-\ln \frac{\alpha \rho_{\nu}}{D}\right) \ln \left(e-\ln \frac{\alpha \rho_{\nu}}{D}\right)}\right] \times \\
& \times\left[1-\frac{1}{e-\ln \frac{\alpha D_{\nu}}{2 D}}-\frac{1}{\left(e-\ln \frac{\alpha D_{\nu}}{2 D}\right) \ln \left(e-\ln \frac{\alpha D_{\nu}}{2 D}\right)}\right]-
\end{aligned}
$$




$$
\begin{gathered}
-\frac{4}{D_{\nu}^{2}}\left[1-\frac{1}{e-\ln \frac{\alpha D_{\nu}}{2 D}}-\frac{1}{\left(e-\ln \frac{\alpha D_{\nu}}{2 D}\right) \ln \left(e-\ln \frac{\alpha D_{\nu}}{2 D}\right)}\right]^{2} \geq \\
\geq\left(\frac{4}{\rho_{\nu} D_{\nu}}-\frac{4}{D_{\nu}^{2}}\right)\left[1-\frac{1}{e-\ln \frac{\alpha D_{\nu}}{2 D}}-\frac{1}{\left(e-\ln \frac{\alpha D_{\nu}}{2 D}\right) \ln \left(e-\ln \frac{\alpha D_{\nu}}{2 D}\right)}\right]^{2} \geq \\
\geq\left(\frac{4}{\rho_{\nu} D_{\nu}}-\frac{4}{D_{\nu}^{2}}\right)\left[1-\frac{1}{e-\ln \frac{\alpha}{2}}-\frac{1}{\left(e-\ln \frac{\alpha}{2}\right) \ln \left(e-\ln \frac{\alpha}{2}\right)}\right]^{2}
\end{gathered}
$$

is valid as well. Here we have used the inequality

$$
e-\ln \frac{\alpha \rho_{\nu}}{D} \geq e-\ln \frac{\alpha D_{\nu}}{2 D}>e
$$

that is obvious since $0<\rho_{\nu} \leqslant \frac{D_{\nu}}{2}$.

For integer $k \geq 0$ we denote

$$
e_{0}=1, \quad e_{k+1}=\exp e_{k} ; \quad \ln _{0} x=x, \quad \ln _{k+1}(x)=\ln \ln _{k}(x) .
$$

Let

$$
f_{k}(t, a)=-\frac{1}{t}+\sum_{i=0}^{k} \frac{1}{t\left(a-\ln \frac{\alpha t}{D}\right) \ln \left(a-\ln \frac{\alpha t}{D}\right) \cdot \ldots \cdot \ln _{i}\left(a-\ln \frac{\alpha t}{D}\right)}, \quad 0<t<D / 2 .
$$

We note that function $f(t)=f_{0}(t, 1)$ was employed in paper [14] in the proof of inequality (5).

We introduce the notation

$$
\varphi_{i}(x, a)=\frac{1}{(a-\ln x) \ln (a-\ln x) \cdot \ldots \cdot \ln _{i}(a-\ln x)} .
$$

Let us show that the function $f_{k}(t, a)$ satisfies the identity

$$
2 f_{k}^{\prime}\left(\rho_{\nu}, e_{k}\right)-f_{k}^{2}\left(\rho_{\nu}, e_{k}\right)=\frac{1}{\rho_{\nu}^{2}}+\sum_{i=0}^{k} \frac{\varphi_{i}^{2}\left(\frac{\alpha \rho_{\nu}}{D}, e_{k}\right)}{\rho_{\nu}^{2}}
$$

and the inequality

$$
2 f\left(\rho_{\nu}, e_{k}\right) f\left(D_{\nu} / 2, e_{k}\right)-f^{2}\left(D_{\nu} / 2, e_{k}\right) \geq\left(\frac{4}{\rho_{\nu} D_{\nu}}-\frac{4}{D_{\nu}^{2}}\right)\left[1-\sum_{i=0}^{k} \varphi_{i}^{2}\left(\frac{\alpha}{2}, e_{k}\right)\right]^{2} .
$$

We prove the first identity by the induction. The case $k=0$ was proven in [14. The proof of the case $k=1$ has been adduced above. Suppose the inequality is valid for all natural numbers less than $k$. Let us show that the statement is valid for the natural number $k+1$.

We observe that

$$
\begin{aligned}
f_{k+1}\left(t, e_{k+1}\right)=f_{k}\left(t, e_{k+1}\right) & +\frac{1}{t\left(e_{k+1}-\ln \frac{\alpha t}{D}\right) \ln \left(e_{k+1}-\ln \frac{\alpha t}{D}\right) \cdot \ldots \cdot \ln _{k+1}\left(e_{k+1}-\ln \frac{\alpha t}{D}\right)}= \\
& =f_{k}\left(t, e_{k+1}\right)+\frac{1}{t} \varphi_{k+1}\left(\frac{\alpha t}{D}, e_{k+1}\right) .
\end{aligned}
$$

Then

$$
\begin{gathered}
2 f_{k+1}^{\prime}\left(\rho_{\nu}, e_{k+1}\right)-f_{k+1}^{2}\left(\rho_{\nu}, e_{k+1}\right)= \\
=2\left(f_{k}\left(t, e_{k+1}\right)+\frac{1}{t} \varphi_{k+1}\left(\frac{\alpha t}{D}, e_{k+1}\right)\right)^{\prime}-\left(f_{k}\left(t, e_{k+1}\right)+\frac{1}{t} \varphi_{k+1}\left(\frac{\alpha t}{D}, e_{k+1}\right)\right)^{2}= \\
=2 f_{k}^{\prime}\left(t, e_{k+1}\right)-f_{k}^{2}\left(t, e_{k+1}\right)+2\left(\frac{1}{t} \varphi_{k+1}\left(\frac{\alpha t}{D}, e_{k+1}\right)\right)^{\prime}-
\end{gathered}
$$




$$
\begin{gathered}
-2 \frac{1}{t} f_{k}\left(t, e_{k+1}\right) \varphi_{k+1}\left(\frac{\alpha t}{D}, e_{k+1}\right)-\frac{1}{t^{2}} \varphi_{k+1}^{2}\left(\frac{\alpha t}{D}, e_{k+1}\right)= \\
=2 f_{k}^{\prime}\left(t, e_{k+1}\right)-f_{k}^{2}\left(t, e_{k+1}\right)-\frac{2 \varphi_{k+1}\left(\frac{\alpha t}{D}, e_{k+1}\right)}{t^{2}}+ \\
+\sum_{i=0}^{k+1} \frac{2 \varphi_{i}\left(\frac{\alpha t}{D}, e_{k+1}\right) \varphi_{k+1}\left(\frac{\alpha t}{D}, e_{k+1}\right)}{t^{2}}+\frac{2 \varphi_{k+1}\left(\frac{\alpha t}{D}, e_{k+1}\right)}{t^{2}}- \\
-\sum_{i=0}^{k} \frac{2 \varphi_{i}^{2}\left(\frac{\alpha t}{D}, e_{k+1}\right) \varphi_{k+1}\left(\frac{\alpha t}{D}, e_{k+1}\right)}{t^{2}}-\frac{\varphi_{k+1}^{2}\left(\frac{\alpha t}{D}, e_{k+1}\right)}{\rho_{\nu}^{2}}= \\
=2 f_{k}^{\prime}\left(t, e_{k+1}\right)- \\
f_{k}^{2}\left(t, e_{k+1}\right)+\frac{1}{\rho_{\nu}^{2}\left(e_{k}-\ln \frac{\alpha \rho_{\nu}}{D}\right)^{2} \ln ^{2}\left(e_{k}-\ln \frac{\alpha \rho_{\nu}}{D}\right) \cdot \ldots \cdot \ln _{k+1}^{2}\left(e_{k}-\ln \frac{\alpha \rho_{\nu}}{D}\right)} .
\end{gathered}
$$

The latter identity leads us to the desired statement.

Inequality (13) can be proven by analogy with the case $k=1$ and with employing inequality

$$
e_{k}-\ln \frac{\alpha \rho_{\nu}}{D} \geq e_{k}-\ln \frac{\alpha D_{\nu}}{2 D}
$$

which is obvious since $0<\rho_{\nu} \leqslant \frac{D_{\nu}}{2}$.

In [14] M. Hoffmann-Ostenhof, T. Hoffmann-Ostenhof, A. Laptev proved that for an arbitrary open set $\Omega \subset \mathbb{R}^{n}$ and an arbitrary function $u \in H_{0}^{1}(\Omega)$ the inequality

$$
\begin{aligned}
& \int_{\Omega}|\nabla u|^{2} d x \geq \frac{n}{4} \int_{\Omega} \int_{\mathbb{S}^{n-1}}\left(2 f^{\prime}\left(\rho_{\nu}(x)\right)-f\left(\rho_{\nu}(x)\right)+\right. \\
& \left.+2 f\left(\rho_{\nu}(x)\right) f\left(D_{\nu}(x) / 2\right)+f^{2}\left(D_{\nu}(x) / 2\right)\right)|u(x)|^{2} d x
\end{aligned}
$$

holds true, where $D \in(0, \infty]$ is the diameter and $f \in \Phi_{2}(0, D / 2)$.

We note that $f\left(t, e_{k}\right) \in \Phi_{2}(0, D / 2)$. Thus, inequality (14) as $f=f\left(t, e_{k}\right)$ gives us the relation

$$
\begin{gathered}
\int_{\Omega}|\nabla u|^{2} d x \geq \frac{n}{4} \int_{\Omega} \int_{\mathbb{S}^{n-1}}\left(\frac{1}{\rho_{\nu}^{2}(x)}+\sum_{i=0}^{k} \frac{\varphi_{i}^{2}\left(\frac{\alpha \rho_{\nu}(x)}{D}, e_{k}\right)}{\rho_{\nu}^{2}(x)}\right) d \omega(\nu)|u(x)|^{2} d x+ \\
+\frac{n}{4}\left[1-\sum_{i=0}^{k} \varphi_{i}^{2}\left(\frac{\alpha}{2}, e_{k}\right)\right]^{2} \int_{\Omega} \int_{\mathbb{S}^{n-1}}\left(\frac{4}{\rho_{\nu}(x) D_{\nu}(x)}-\frac{4}{D^{2}(x)_{\nu}}\right) d \omega(\nu)|u(x)|^{2} d x .
\end{gathered}
$$

In [14] the authors also showed that

$$
\int_{\mathbb{S}^{n-1}} \frac{4}{\rho_{\nu}(x) D_{\nu}(x)}-\frac{4}{D_{\nu}^{2}(x)} d \omega(\nu) \geq\left(\frac{s_{n-1}}{n}\right)^{2 / n} \frac{1}{\left|\Omega_{x}\right|^{2 / n}} .
$$

Combining two latter inequalities, we obtain

$$
\begin{aligned}
\int_{\Omega}|\nabla u|^{2} d x & \geq \frac{n}{4} \int_{\Omega} \int_{\mathbb{S}^{n-1}}\left(\frac{1}{\rho_{\nu}^{2}(x)}+\sum_{i=0}^{k} \frac{\varphi_{i}^{2}\left(\frac{\alpha \rho_{\nu}(x)}{D}, e_{k}\right)}{\rho_{\nu}^{2}(x)}\right) d \omega(\nu)|u(x)|^{2} d x+ \\
+ & {\left[1-\sum_{i=0}^{k} \varphi_{i}^{2}\left(\frac{\alpha}{2}, e_{k}\right)\right]^{2} \frac{n^{(n-2) / 2}}{4} s_{n-1}^{2 / n} \int_{\Omega} \frac{|u(x)|^{2}}{\left|\Omega_{x}\right|^{2 / n}} d x . }
\end{aligned}
$$

Suppose now that $\Omega \subset \mathbb{R}^{n}$ is a regular domain with the constant of regularity $c$. In [21] A.M. Tukhvatullina showed that then

$$
\int_{\mathbb{S}^{n-1}} \frac{d \omega(\nu)}{\rho_{\nu}^{2}(x)} \geq \frac{2}{c^{2} \delta^{2}(x)}, \forall x \in \Omega .
$$


We observe that

$$
\begin{aligned}
& \int_{\mathbb{S}^{n-1}}\left(\frac{1}{\rho_{\nu}^{2}(x)}+\sum_{i=0}^{k} \frac{\varphi_{i}^{2}\left(\frac{\alpha \rho_{\nu}(x)}{D}, e_{k}\right)}{\rho_{\nu}^{2}(x)}\right) d \omega(\nu) \geq \\
& \geq\left(1+\sum_{i=0}^{k} \varphi_{i}^{2}\left(\frac{\alpha \rho_{\nu}(x)}{D}, e_{k}\right)\right) \int_{\mathbb{S}^{n-1}} \frac{d \omega(\nu)}{\rho_{\nu}^{2}(x)} .
\end{aligned}
$$

Hence,

$$
\begin{gathered}
\int_{\mathbb{S}^{n-1}}\left(\frac{1}{\rho_{\nu}^{2}(x)}+\sum_{i=0}^{k} \frac{\varphi_{i}^{2}\left(\frac{\alpha \rho_{\nu}(x)}{D}, e_{k}\right)}{\rho_{\nu}^{2}(x)}\right) d \omega(\nu) \geq \\
\geq\left(1+\sum_{i=0}^{k} \varphi_{i}^{2}\left(\frac{\alpha \rho_{\nu}(x)}{D}, e_{k}\right)\right) \frac{2}{c^{2} \delta^{2}(x)} .
\end{gathered}
$$

Thus, we arrive at the following result.

Theorem 5. Suppose $\Omega \subset \mathbb{R}^{n}$ is a regular domain with the constant of regularityc and $0<\alpha \leqslant 2$. Then for an arbitrary function $u \in C_{0}^{\infty}(\Omega)$ and $k \in \mathbb{N}$ the inequality

$$
\begin{aligned}
& \int_{\Omega}|\nabla u|^{2} d x \geq \frac{n}{2 c^{2}} \int_{\Omega} \frac{|u(x)|^{2}}{\delta^{2}(x)}\left(1+\sum_{i=0}^{k} \varphi_{0}^{2}\left(\frac{\alpha \delta}{D}, e_{k}\right) \cdot \ldots \cdot \varphi_{i}^{2}\left(\frac{\alpha \delta}{D}, e_{k}\right)\right) d x+ \\
& +\left[1-\sum_{i=0}^{k} \varphi_{0}\left(\frac{\alpha}{2}, e_{k}\right) \cdot \ldots \cdot \varphi_{i}\left(\frac{\alpha}{2}, e_{k}\right)\right]^{2} \frac{n^{(n-2) / 2}}{4} s_{n-1}^{2 / n} \frac{1}{|\Omega|^{2 / n}} \int_{\Omega}|u(x)|^{2} d x
\end{aligned}
$$

holds true, where

$$
\varphi_{i}\left(x, e_{k}\right)=\frac{1}{\left(e_{k}-\ln x\right) \ln \left(e_{k}-\ln x\right) \cdot \ldots \cdot \ln _{i}\left(e_{k}-\ln x\right)}, i \leqslant k .
$$

We express our sincere gratitude to our supervisor, professor Farit Gabidinovich Avkhadiev, for useful advices and valuable remarks.

\section{BIBLIOGRAPHY}

1. Yu.A. Dubinski. A Hardy-type inequality and its applications. // Trudy MIAN. 2010. V. 269. P. 112-132. [Proc. Steklov Inst. Math. 2010. V. 269, No. 1. P. 106-126.]

2. A. Balinsky, A. Laptev, A.V. Sobolev. Generalized Hardy inequality for the magnetic Dirichlet forms. // J. Stat. Phys. 2004. V. 116, No. 4. P. 507-521.

3. A. Laptev, T. Weidl. Hardy inequalities for magnetic Dirichlet forms. // Oper. Theory. Adv. Appl. 1999. V. 108. P. 299-305.

4. M. Solomyak. A remark on the Hardy inequalities. // Integr. Equat. Oper. Th. 1994. V. 19, No. 1. P. $120-124$.

5. E.B. Davies. Spectral Theory and Differential Operators. Cambridge Studies in Advanced Mathematics. V. 42. Cambridge Univ.Press, Cambridge. 51995.

6. E.B. Davies. A Review of Hardy Inequalities. // Oper. Theory. Adv. Appl. 1999. V. 110. P. 55-67.

7. F.G. Avkhadiev, K.-J. Wirths. Sharp Hardy-type inequalities with Lamb's constants. // Bull. Belg. Math. Soc. 2011. V. 18, No. 4. P. 723-736.

8. F.G. Avkhadiev. Inequalities for integral characteristics of domains. Kazan, KSU. 2006. (in Russian.)

9. F.G. Avkhadiev. Hardy type inequalities in higher dimensions with explicit estimate of constants // Lobachevskii J. Math. 2006. V. XXI. P. 3-31.

10. F.G. Avkhadiev, K.-J. Wirths. Unified Poincaré and Hardy inequalities with sharp constants for convex domains. // Z. Angew. Math. Mech. 2007. V. 87, No. 8-9. P. 632-642. 
11. F.G. Avkhadiev. Hardy-type inequalities on planar and spatial open sets. // Trudy MIAN. 2006. V. 255. P. 8-18. [Proc. Steklov Inst. Math. 2006. V. 255, No. 1. P. 2-12.]

12. F.G. Avkhadiev, R.G. Nasibullin, I.K. Shafigullin. Hardy-type inequalities with power and logarithmic weights in domains of the Euclidean space. // Izv. VUZov. Matem. 2011. No. 9. P. 90-94. [Russ. Math. (Izvestiya VUZ. Matematika). 2011. V. 55, No. 9. P. 76-79.]

13. H. Brezis, M. Marcus. Hardy's inequality revisited. // Ann. Scuola Norm. Sup. Pisa Cl. Sci. 1997. V. 25, No. 1-2. P. 217-237.

14. M. Hoffmann-Ostenhof, T. Hoffmann-Ostenhof, A. Laptev. A geometrical version of Hardy's inequality. // J. Funct. Anal. 2002. V. 189, No. 2. P. 539-548.

15. J. Tidblom. A geometrical version of Hardy's inequality for $W_{0}^{1, p}(\Omega)$. // Proc. Amer. Math. Soc. 2004. V. 132, No. 8. P. 2265-2271.

16. G. Barbatis, S. Filippas, A. Tertikas. Refined geometric $L^{p}$ Hardy inequalities. // Comm. Contem. Math. 2003. V. 5, No. 6. 2003. P. 869-881.

17. M. Del Pino, J. Dolbeault, S. Filippas and A. Tertikas A logarithmic Hardy inequality // J. Funct. Anal. 259. 2010. P. 2045-2072.

18. S.M. Buckley, R. Hurri-Syrjänene Iterated log-scale Orlicz-Hardy inequalities. // Preprint. Department of Mathematics, National University of Ireland, Maynooth. 2011.

19. R.G. Nasibullin. Hardy type inequalities invovling iterated logarithms // Trudy matem. centra im. N.I. Lobachevskogo. 2011. V. 43. P. 262-263. (in Russian.)

20. R.G. Nasibullin. A generalization of Hardy inequality. // Trudy matem. centra im. N.I. Lobachevskogo. V. 44. P. 221-222. (in Russian.)

21. A.M. Tukhvatullina. Hardy type inequalities for a special family of non-convex domains. // Uchenye zapiski Kazan. gos. univ. Ser. fiz.-mat. nauki. 2011. V. 153, No. 1. P. 211-220. (in Russian.)

22. A.M. Tukhvatullina. Generalization of regularity criterion for Daives domain for multidimensional domains and its application in Hardy type inequalities. // Trudy matem. centra im. N.I. Lobachevskogo. 2011. V. 43. P. 350-351. (in Russian.)

23. A.M. Tukhvatullina. Sufficient condition for regularity of domain and its application in Hardy type inequalities. // Trudy matem. centra im. N.I. Lobachevskogo. 2009. V. 38. P. 285-287. (in Russian.)

Ramil Gaisaevich Nasibullin,

Kazan (Volga Region) Federal University,

Kremlevskya str., 18,

420008, Kazan, Russia

E-mail: NasibullinRamil@gmail.com

Alina Mikhailovna Tukhvatullina,

Kazan (Volga Region) Federal University,

Kremlevskya str., 18,

420008, Kazan, Russia

E-mail: kzn.alina@gmail.com 